[8] J. L. Sullivan and J. W. Adams, "PCLS IIR digital filters with simultaneous frequency response magnitude and group delay specifications," IEEE Trans. Signal Processing, vol. 46, pp. 2853-2861, Nov. 1998.

[9] A. T. Chottera and G. A. Jullien, "A linear programming approach to recursive filter design with linear phase," IEEE Trans. Circuits Syst., vol. 29, pp. 139-149, Mar. 1982.

[10] M. C. Lang, "Weighted least squares IIR filter design with arbitrary magnitude and phase responses and specified stability margin," in IEEE Symp. Advances in Digital Filtering and Signal Processing, Victoria, B.C., Canada, 1998, pp. 82-86.

[11] P. A. Regalia, Adaptive IIR Filtering in Signal Processing and Control. New York: Marcel Dekker, 1995.

[12] X. Chen and T. W. Parks, "Design of FIR filters in the complex domain," IEEE Trans. Acoust., Speech, Signal Processing, vol. 35, pp. 144-153, Feb. 1987.

[13] H. Xu, W. S. Lu, and A. Antoniou, "Improved iterative methods for the design of quadrature mirror-image filter bands," IEEE Trans. Circuits Syst. II, vol. 43, pp. 363-371, May 1996

\section{Robust D-Stability Analysis for Uncertain Discrete Singular Systems With State Delay}

\author{
Shengyuan $\mathrm{Xu}$, James Lam, and Liqian Zhang
}

\begin{abstract}
This brief investigates the problem of robust D-stability analysis for uncertain discrete singular systems with state delay and structured uncertainties. Sufficient conditions are developed to ensure that, when the nominal discrete singular delay system is regular, causal and all its finite poles are located within a specified disk, the uncertain system still preserves all these properties when structured uncertainties are added into the nominal system. A computationally simple approach is proposed and a numerical example is given to demonstrate the application of the proposed method.
\end{abstract}

Index Terms-Discrete systems, robust D-stability, singular systems, state delay, structured uncertainties.

\section{INTRODUCTION}

It is well known that satisfactory transient behavior of a linear timeinvariant control system can be achieved by using the pole assignment technique. When there is no uncertainty in the system, it is possible to exactly place the poles at specified locations. However, in practical applications, one cannot place all the poles in precise locations due to parametric uncertainties originating from various sources, such as identification errors, aging of elements, and so on. Therefore, it is reasonable in practice to assign all the poles of the closed-loop system in a desired region rather than exact assignment. This has brought about

Manuscript received March 20, 2001; revised August 21, 2001. The work of $\mathrm{S}$. Xu was supported in part by the Belgium Programme on Inter-university Poles of Attraction, initiated by the Belgian State, Prime Minister's Office for Science, Technology and Culture. The work of J. Lam and L. Zhang was supported by Hong Kong University under Grant CRCG 10203300/19870. The scientific responsibility rests with its authors. This paper was recommended by Associate Editor X. Yu.

$\mathrm{S}$. Xu is with the Center for Systems Engineering and Applied Mechanics (CESAME), Université catholique de Louvain, Batiment Euler, Belgium (e-mail: xu@auto.ucl.ac.be).

J. Lam and L. Zhang are with the Department of Mechanical Engineering, University of Hong Kong, Pokfulam Road, Hong Kong.

Publisher Item Identifier S 1057-7122(02)03129-X. the study of pole assignment in a specified region, particular circular region in the left half complex plane for continuous systems or in the unit disk with center at the origin for discrete systems. The problem of assigning all poles of a system in a specified disk is referred to as a D-pole placement problem. Considerable amount of results on this issue have been reported in the literature, see, e.g., [3], [9], [10]. It is worth mentioning that these results have been further extended to discrete systems with parameter uncertainties and time delays [12], [16], [17].

Singular systems have been studied by many researchers in the past years. This is due not only to theoretical interest but also to the extensive applications of these systems to circuits [14], boundary control systems [15], chemical processes [11], and other areas. Singular systems are also referred to as descriptor systems, implicit systems, generalized state-space systems, differential-algebraic systems or semi-state systems [5], [13]. The problem of pole assignment for singular systems has also received considerable attention. Under certain controllability assumptions and with different treatments, the exact pole assignment problem for singular systems have been dealt with [4], [8]. When parametric uncertainties appear, the problems of robust D-stability analysis and robust D-pole placement for discrete-time singular systems have been investigated in [7], and sufficient conditions have been obtained. Similar results for continuous-time singular systems have been reported in [6]. Both results in [6] and [7] can be viewed as extensions of those for state-space systems. It is worth pointing out that the robust D-stability problem for singular systems is much more complicated than that for state-space systems because it requires to consider not only D-stability robustness, but also regularity and impulse immunity (for continuous singular systems) and causality (for discrete singular systems) simultaneously [6], [7], while for state-space systems, the latter two issues do not arise. It is also noted that when both parametric uncertainties and state delay appear in singular discrete systems, no results on D-stability robustness for these systems are available in the literature, this problem is still open and remains challenging.

In this brief, we consider the problem of robust D-stability analysis for uncertain discrete singular systems with state delay. Structured uncertainties are discussed. The purpose of this paper is to develop conditions such that, when the nominal discrete singular delay system is regular, causal and all its finite poles are located within a specified disk, the uncertain system still preserves all these properties when structured uncertainties are present in the nominal system. We first propose a computationally simple approach, which can be viewed as an extension of that in [3], to present a robust stability result for uncertain discrete singular delay systems. Then, based on this, sufficient conditions for D-stability robustness are obtained. Finally, a numerical example is given to demonstrate the results.

Notation: Throughout this paper, for matrices $X, Y \in \mathbb{R}^{n \times n}$, the notation $X \geq Y$ means that $X_{i j} \geq Y_{i j}, i, j=1,2, \ldots n$, where $X_{i j}, Y_{i j}(i, j=1,2, \ldots n)$, are elements of $X$ and $Y$, respectively. $I$ is the identity matrix with appropriate dimension. The superscript " $T$ " represents the transpose. $D_{\text {int }}(q, r)$ stands for the interior of the disk with center at $q+j 0$ and radius $r$. Thus, $D_{\text {int }}(0,1)$ is the interior of the unit disk with center at the origin, and for stability, we require $D_{\text {int }}(q, r) \subset D_{\text {int }}(0,1) . \sigma\left(E, A, A_{d}\right)=\{z \mid \operatorname{det}(z E-A-$ $\left.\left.z^{-d} A_{d}\right)=0\right\} \cdot \sigma(E, A)=\{z \mid \operatorname{det}(z E-A)=0\} .|x|$ denotes the Euclidean norm if $x$ is a vector and absolute value if $x$ is a scalar. $\rho(M)$ refers to spectral radius of matrix $M$ and $|M|_{m}$ is the modulus matrix of $M$. Matrices, if not explicitly stated, are assumed to have compatible dimensions. 


\section{PRELIMINARIES AND PROBLEM FORMULATION}

Consider the following uncertain linear discrete singular system with state delay:

$$
E x(k+1)=(A+\Delta A) x(k)+\left(A_{d}+\Delta A_{d}\right) x(k-d)
$$

where $x(k) \in \mathbb{R}^{n}$ is the state and the matrix $E \in \mathbb{R}^{n \times n}$ may be singular, we shall assume that rank $E=r \leq n . A$ and $A_{d}$ are known real constant matrices with appropriate dimensions. $d>0$ is a known positive integer time delay of the system, $\Delta A$ and $\Delta A_{d}$ are highly structured matrices representing time-invariant parameter uncertainties, and are assumed to have the following properties [7], [12]:

$$
|\Delta A|_{m} \leq M_{A} \quad\left|\Delta A_{d}\right|_{m} \leq M_{d}
$$

where $M_{A}$ and $M_{d}$ are constant matrices whose elements are all nonnegative. $\Delta A$ and $\Delta A_{d}$ are said to be admissible if (2) holds.

The nominal discrete singular delay system of (1) is given as follows:

$$
E x(k+1)=A x(k)+A_{d} x(k-d) .
$$

Throughout this paper, we use the following definitions.

Definition 1 [5], [18]:

I) The pair $(E, A)$ is said to be regular if $\operatorname{det}(z E-A)$ is not identically zero.

II) The pair $(E, A)$ is said to be causal if $\operatorname{deg}(\operatorname{det}(z E-A))=$ rank $E$.

III) The singular system $E x(k+1)=A x(k)$ or simply the pair $(E, A)$ is said to be stable if $\sigma(E, A) \subset D_{\text {int }}(0,1)$.

Definition 2 [1]: For a regular pair $(E, A), \sigma(E, A)$ are said to be finite spectrum (or finite modes) of the pair $(E, A)$. Suppose a nonzero vector $\nu_{1} \in \mathbb{R}^{n}, \nu_{1} \neq 0$ satisfying $E \nu_{1}=0$. Then, the infinite eigenvalues associated with the generalized principal vectors $\nu_{k}$ satisfying $\nu_{k} \neq 0, E \nu_{k}=A \nu_{k-1}, k=2,3,4, \ldots$, are said to be the noncausal modes.

Proposition 1 [1]: The following statements are equivalent.

I) The pair $(E, A)$ is causal;

II) The pair $(E, A)$ has no noncausal modes;

III) $\quad(z E-A)^{-1}$ is proper.

For the discrete singular delay system (3), if we set

$$
X(k)=\left[x(k)^{T}, x(k-1)^{T}, \ldots, x(k-d)^{T}\right]^{T}
$$

then system (3) can be rewritten as

$$
\hat{E} X(k+1)=\hat{A} X(k)
$$

where

$$
\begin{aligned}
& \hat{E}=\left[\begin{array}{c|c}
E & 0_{n \times n d} \\
\hline 0_{n d \times n} & I_{n d \times n d}
\end{array}\right] \\
& \hat{A}=\left[\begin{array}{cc|c}
A & 0_{n \times n(d-1)} & A_{d} \\
\hline I_{n d \times n d} & 0_{n d \times n}
\end{array}\right] .
\end{aligned}
$$

Therefore, we introduce the following definition for discrete singular delay system (3).

Definition 3: The discrete singular delay system (3) is said to be regular if system (4) is regular. System (3) is said to be causal if system (4) is causal. System (3) is said to be stable if system (4) is stable.
Remark 1: In the case when singular system (3) is regular, causal and stable, from Definition 3, it can be shown that for any admissible initial condition, the following singular system:

$$
E x(k+1)=A x(k)+A_{d} x(k-d)+u(k)
$$

has a unique solution, and its solution can be determined by the initial condition and past inputs, no future inputs is required. That is, $x(k)$ $(k \geq 0)$ can be completely determined at any time $k$ by $x(0)$ and past inputs $u(0), u(1), \ldots, u(k)$. Furthermore, when the input $u(k) \equiv 0$, that is, (6) reduces to (3), in this case, its solution $x(k)$ satisfies $x(k) \rightarrow$ $0, k \rightarrow \infty$.

The robust D-stability problem to be dealt with in this paper can be formulated as follows. Suppose the nominal discrete singular delay system (3) is regular, causal and its finite eigenvalues are located within disk $D_{\text {int }}(q, r)$, that is, $\sigma(\hat{E}, \hat{A}) \subset D_{\text {int }}(q, r)$, we shall develop sufficient conditions guaranteeing that the uncertain discrete singular delay system (1) remains regular, causal and its finite eigenvalues are located within disk $D_{\text {int }}(q, r)$, that is, $\sigma(\hat{E}, \hat{A}+\Delta \hat{A}) \subset D_{\text {int }}(q, r)$, where

$$
\Delta \hat{A}=\left[\begin{array}{cc|c}
\Delta A & 0_{n \times n(d-1)} & \Delta A_{d} \\
\hline 0_{n d \times n d} & 0_{n d \times n}
\end{array}\right] .
$$

\section{MAIN RESUlTS}

In this section, we first present a computationally simple sufficient condition ensuring robust stability for uncertain discrete singular delay systems, which will play a key role in the derivation of robust D-stability results.

From Definition 3, the regularity, causality and stability of the uncertain singular delay system (1) can be determined by testing whether the pair $(\hat{E}, \hat{A}+\Delta \hat{A})$ is regular, causal as well as stable or not, for which many testing conditions exist in the literature [5], [13]. However, it should be noted that for a $\Delta d$ increase in the time delay $d$ the sizes of matrix $\hat{E}$ and $\hat{A}+\Delta \hat{A}$ will increase by $n \Delta d$. Therefore, for large delays the sizes of matrix $\hat{E}$ and $\hat{A}+\Delta \hat{A}$ become large and the testing conditions for the pair $(\hat{E}, \hat{A}+\Delta \hat{A})$ will become difficult to apply. It is then desirable to develop simple conditions to test the regularity, causality and stability of discrete singular delay system (1).

The following lemma shows that testing the regularity, causality and stability of system (3) can be converted into testing the regularity and causality of the pair $(E, A)$ together with $\sigma\left(E, A, A_{d}\right) \subset D_{\text {int }}(0,1)$.

Lemma 1: The discrete singular delay system (3) is regular, causal and stable if and only if the pair $(E, A)$ is regular, causal and

$$
\sigma\left(E, A, A_{d}\right) \subset D_{\text {int }}(0,1) .
$$

Proof: (Sufficiency) Suppose the pair $(E, A)$ is regular, causal and $\sigma\left(E, A, A_{d}\right) \subset D_{\text {int }}(0,1)$, then it follows from [5] that there exist two invertible matrices $M$ and $N$ such that

$$
M E N=\left[\begin{array}{ll}
I & 0 \\
0 & 0
\end{array}\right] \quad M A N=\left[\begin{array}{cc}
A_{1} & 0 \\
0 & I
\end{array}\right] .
$$

Writing

$$
M A_{d} N=\left[\begin{array}{ll}
A_{d 1} & A_{d 2} \\
A_{d 3} & A_{d 4}
\end{array}\right]
$$

compatible with (8) and noting

$$
\operatorname{det}(z \hat{E}-\hat{A})=z^{n d} \operatorname{det}\left(z E-A-z^{-d} A_{d}\right)
$$


we have

$$
\begin{aligned}
\operatorname{det}(z \hat{E}-\hat{A})=z^{n d} & \operatorname{det}\left(M^{-1} N^{-1}\right) \\
& \cdot \operatorname{det}\left[\begin{array}{cc}
z I-A_{1}-z^{-d} A_{d 1} & -z^{-d} A_{d 2} \\
-z^{-d} A_{d 3} & -I-z^{-d} A_{d 4}
\end{array}\right] .
\end{aligned}
$$

It is easy to show that for sufficiently large $z, \operatorname{det}(z \hat{E}-\hat{A}) \neq 0$. Therefore, there exists a scalar $z \in \mathbb{C}$ such that $\operatorname{det}(z \hat{E}-\hat{A}) \neq 0$, this implies that the pair $(\hat{E}-\hat{A})$ is regular. Furthermore, from (10), it can be seen that

$$
\operatorname{deg}(\operatorname{det}(z \hat{E}-\hat{A}))=n d+\operatorname{rank} E=\operatorname{rank} \hat{E}
$$

Hence, it follows from Definition 1 that the pair $(\hat{E}, \hat{A})$ is causal. The stability of the pair $(\hat{E}-\hat{A})$ can be shown from (7) and (9). Thus, from Definition 3, the discrete singular delay system (3) is regular, causal and stable.

(Necessity) Suppose the discrete singular delay system (3) is regular, causal and stable. Then from Definition 3, we have that the pair $(\hat{E}, \hat{A})$ is regular, causal and stable. Next we shall show that the pair $(E, A)$ is regular, causal and $\sigma\left(E, A, A_{d}\right) \subset D_{\text {int }}(0,1)$. Suppose, by contradiction, the pair $(E, A)$ is not regular, then from [5, Theorem 1-2.1], there exist an integer $k \geq 1$ and a vector $\xi^{T}=\left[\xi_{1}^{T}, \xi_{2}^{T}, \ldots, \xi_{k}^{T}\right]$, $\xi \neq 0, \xi_{i} \in \mathbb{R}^{n}, i=1,2, \ldots, k$, such that

$$
\Gamma(k) \xi=0
$$

where

$$
\Gamma(k)=\left[\begin{array}{cccc}
E & & & \\
A & E & & \\
& A & \ddots & \\
& & \ddots & E \\
& & & A
\end{array}\right] \in \mathbb{R}^{(k+1) n \times n k} .
$$

Without loss of generality, suppose $\xi_{1} \neq 0$, then it follows from (11) that

$$
E \xi_{1}=0, \quad A \xi_{1}=-E \xi_{2} .
$$

Let

$$
\begin{gathered}
v_{1}^{T}=\left[\xi_{1}^{T}, 0,0, \ldots, 0\right] \quad v_{2}^{T}=\left[-\xi_{2}^{T}, \xi_{1}^{T}, 0, \ldots, 0\right], \\
v_{1}, v_{2} \in \mathbb{R}^{(d+1) n}
\end{gathered}
$$

then $v_{1} \neq 0, v_{2} \neq 0$. From (12), it is easy to see

$$
\hat{E} v_{1}=0, \quad \hat{E} v_{2}=\hat{A} v_{1} .
$$

From Proposition 1, we have that (13) is a contradiction because the pair $(\hat{E}, \hat{A})$ is causal. Therefore, the pair $(E, A)$ is regular.

Now, we show the pair $(E, A)$ is causal. Suppose, by contradiction, the pair $(E, A)$ is not causal. Then, it follows from Proposition 1 that there exist at least two nonzero vectors $\nu_{1}, \nu_{2} \in \mathbb{R}^{n}$ satisfying $E \nu_{1}=0$ and $E \nu_{2}=A \nu_{1}$. Set

$$
\begin{gathered}
\eta_{1}=\left[\nu_{1}^{T}, 0,0 \cdots 0\right]^{T} \quad \eta_{2}=\left[\nu_{2}^{T}, \nu_{1}^{T}, 0, \ldots 0\right]^{T}, \\
\eta_{1}, \eta_{2} \in \mathbb{R}^{(d+1) n} .
\end{gathered}
$$

It is easy to show that

$$
\hat{E} \eta_{1}=0, \quad \hat{E} \eta_{2}=\hat{A} \eta_{1} .
$$

This is also a contradiction because the pair $(\hat{E}, \hat{A})$ is causal. Therefore, the pair $(E, A)$ is causal.

Finally, $\sigma\left(E, A, A_{d}\right) \subset D_{\text {int }}(0,1)$ can be established by noting the stability of the pair $(\hat{E}, \hat{A})$ and the equality (9). This completes the proof.

Before stating the robust stability results, we introduce the following lemmas which will be used in the proof of our main results.

Lemma 2 [2]: Let $M(z)$ be a square rational matrix and be decomposed uniquely as $M(z)=M_{p}(z)+M_{s p}(z)$, where $M_{p}(z)$ is a polynomial matrix and $M_{s p}(z)$ is a strictly proper rational matrix. Then, $M^{-1}(z)$ is proper if and only if $M_{p}^{-1}(z)$ exists and is proper.

Lemma 3: Suppose the nominal discrete singular delay system (3) is regular, causal and stable, and define $G_{p}(z)$ as

$$
G_{p}(z)=\left(z E-A-A_{d} z^{-d}\right)^{-1}
$$

then

$$
\left|G_{p}(z)\right|_{m} \leq H, \quad|z| \geq 1
$$

where

$$
H=\sum_{k=0}^{\infty}|G(k)|_{m}, \quad|z| \geq 1
$$

and $G(k)$ is the pulse response sequence matrix of $G_{p}(z)$.

Proof: Since $G(k)$ is the pulse response sequence matrix of $G_{p}(z)$, we can write

$$
G_{p}(z)=\left(z E-A-A_{d} z^{-d}\right)^{-1}=\sum_{k=0}^{\infty} G(k) z^{-k} .
$$

Then, by [7, Lemma 3], the desired result follows immediately.

Now, we are in a position to present the robust stability result for uncertain discrete singular delay systems.

Theorem 1: Suppose the nominal discrete singular delay system (3) is regular, causal and stable. Then, for all admissible uncertainties, the uncertain discrete singular delay system (1) remains regular, causal and stable if

$$
\rho\left(H\left(M_{A}+M_{d}\right)\right)<1
$$

where $H$ is defined in (15).

Proof: From Lemma 1, the regularity, causality, and stability of system (3) imply that the pair $(E, A)$ is regular and causal and $\sigma\left(E, A, A_{d}\right) \subset D_{\text {int }}(0,1)$. Therefore, for $|z| \geq 1$, we have

$$
\operatorname{det}\left(z E-A-A_{d} z^{-d}\right) \neq 0 \text {. }
$$

Using [7, Lemma 3] and noting (17), it follows that

$\rho\left(\left(z E-A-A_{d} z^{-d}\right)^{-1} \Delta A\right) \leq \rho\left(H M_{A}\right) \leq \rho\left(H\left(M_{A}+M_{d}\right)\right)<1$

for $|z| \geq 1$. Thus, for $|z| \geq 1$

$$
\operatorname{det}\left(I-\left(z E-A-A_{d} z^{-d}\right)^{-1} \Delta A\right) \neq 0 .
$$

Considering the regularity and causality of the pair $(E, A)$ and noting (18), we can show that there exists a sufficiently large scalar $z_{1} \in \mathbb{C}$ such that

$$
\operatorname{det}\left[I-\left(z_{1} E-A-A_{d} z_{1}^{-d}\right)^{-1}\left(\Delta A-A_{d} z_{1}^{-d}\right)\right] \neq 0
$$

and

$$
\operatorname{det}\left(z_{1} E-A-A_{d} z_{1}^{-d}\right) \neq 0
$$


Then, it follows from (19) and (20) that

$$
\begin{aligned}
& \operatorname{det}\left[z_{1} E-(A+\Delta A)\right] \\
& =\operatorname{det}\left[\left(z_{1} E-A-A_{d} z_{1}^{-d}\right)-\left(\Delta A-A_{d} z_{1}^{-d}\right)\right] \\
& =\operatorname{det}\left(z_{1} E-A-A_{d} z_{1}^{-d}\right) \\
& \quad \cdot \operatorname{det}\left[I-\left(z_{1} E-A-A_{d} z_{1}^{-d}\right)^{-1}\left(\Delta A-A_{d} z_{1}^{-d}\right)\right] \neq 0
\end{aligned}
$$

which implies that the pair $(E, A+\Delta A)$ is regular for all admissible uncertainties. Next, we show that, for all admissible uncertainties, the pair $(E, A+\Delta A)$ is causal. To this end, we note that the matrix ( $z E-$ $\left.A-A_{d} z^{-d}\right)^{-1}$ can be expressed as

$$
\left(z E-A-A_{d} z^{-d}\right)^{-1}=G(0)+G_{s p}(z)
$$

where $G(0)$ is a constant matrix and $G_{s p}(z)$ is a strictly proper rational matrix. From [7, Lemma 3] and (17), it is easy to see that

$$
\begin{aligned}
\rho(G(0) \Delta A) & \leq \rho\left(|G(0)|_{m} M_{A}\right) \leq \rho\left(H M_{A}\right) \\
& \leq \rho\left(H\left(M_{A}+M_{d}\right)\right)<1 .
\end{aligned}
$$

This inequality implies that $I-G(0) \Delta A$ is invertible. We write

$$
\begin{aligned}
& {[z E-(A+\Delta A)]^{-1}} \\
& =\left[z E-(A+\Delta A)-A_{d} z^{-d}+A_{d} z^{-d}\right]^{-1} \\
& =[ \\
& \quad\left[-\left(z E-A-A_{d} z^{-d}\right)^{-1} \Delta A\right. \\
& \left.\quad+\left(z E-A-A_{d} z^{-d}\right)^{-1} A_{d} z^{-d}\right]^{-1} \\
& \quad \cdot\left(z E-A-A_{d} z^{-d}\right)^{-1} \\
& =\left[(I-G(0) \Delta A)+\left(z E-A-A_{d} z^{-d}\right)^{-1} A_{d} z^{-d}\right. \\
& \left.\quad \quad-G_{s p}(z) \Delta A\right]^{-1}\left(z E-A-A_{d} z^{-d}\right)^{-1} .
\end{aligned}
$$

Noting $\left(z E-A-A_{d} z^{-d}\right)^{-1} A_{d} z^{-d}-G_{s p}(z) \Delta A$ is a strictly proper rational matrix and recalling $I-G(0) \Delta A$ is invertible, it then follows from Lemma 2 that $\left[(I-G(0) \Delta A)+\left(z E-A-A_{d} z^{-d}\right)^{-1} A_{d} z^{-d}-\right.$ $\left.G_{s p}(z) \Delta A\right]^{-1}$ is proper and so is $(z E-(A+\Delta A))^{-1}$. Hence, by Proposition 1, we have that the pair $(E, A+\Delta A)$ is causal.

Now, using [7, Lemma 3] again, we obtain

$$
\rho\left[\left(z E-A-A_{d} z^{-d}\right)^{-1}\left(\Delta A+\Delta A_{d}\right)\right] \leq \rho\left(H\left(M_{A}+M_{d}\right)\right)<1
$$

for $|z| \geq 1$. Therefore, it can be shown that

$$
\begin{aligned}
& \operatorname{det}\left(z E-(A+\Delta A)-\left(A_{d}+\Delta A_{d}\right) z^{-d}\right) \\
& =\operatorname{det}\left(z E-A-A_{d} z^{-d}\right) \\
& \quad \cdot \operatorname{det}\left(I-\left(z E-A-A_{d} z^{-d}\right)^{-1}\left(\Delta A+\Delta A_{d}\right)\right) \neq 0
\end{aligned}
$$

for $|z| \geq 1$. That is,

$$
\rho\left(E, A+\Delta A, A_{d}+\Delta A_{d}\right) \subset D_{\text {int }}(0,1) .
$$

Finally, by recalling the pair $(E, A+\Delta A)$ is regular and causal, noting (23) and using Lemma 1, we have that the uncertain discrete singular delay system (1) is regular, causal and stable for all admissible uncertainties. This completes the proof.

The following result is needed in the proof of the main results.

Lemma 4: The discrete singular delay system (3) is regular, causal and all its finite eigenvalues are located within the disk $D_{\text {int }}(q, r)$ if and only if the pair $\left(E, A_{r}\right)$ is regular, causal and

$$
\operatorname{det}\left(v E-A_{r}-\frac{1}{r}(r v+q)^{-d} A_{d}\right) \neq 0, \quad|v| \geq 1
$$

where

$$
A_{r}=\frac{1}{r}(A-q E) \text {. }
$$

Proof: From Lemma 1 and (9), it follows that (3) is regular, causal and all its finite eigenvalues are located within disk $D_{\text {int }}(q, r)$ if and only if the pair $(E, A)$ is regular, causal and $\sigma\left(E, A, A_{d}\right) \subset D_{\text {int }}(q, r)$, or

$$
\operatorname{det}\left(z E-A-z^{-d} A_{d}\right) \neq 0, \quad\left|\frac{z-q}{r}\right| \geq 1
$$

Let

$$
v=\frac{z-q}{r} .
$$

Then, we have that the pair $(E, A)$ is regular, causal and (26) holds if and only if the pair $\left(E, A_{q}\right)$ is regular, causal and (24) holds.

Now, we present the robust D-stability result for uncertain singular delay system (1) in the following theorem.

Theorem 2: Suppose the nominal discrete singular delay system (3) is regular, causal and all its finite eigenvalues are located within the disk $D_{\text {int }}(q, r)$. Then, for all admissible uncertainties, the uncertain discrete singular delay system (1) remains regular, causal and all its finite eigenvalues are located within the disk $D_{\text {int }}(q, r)$ if

$$
\rho\left(r^{-1} H_{r}\left(M_{A}+\delta^{-d} M_{d}\right)\right)<1
$$

where

$$
\delta=\min (|q+r|,|q-r|)
$$

and $H_{r}$ is defined as

$$
H_{r}=\sum_{k=0}^{\infty}\left|G_{r}(k)\right|_{m}
$$

where $G_{r}(k)$ is the pulse response sequence matrix of $G_{p r}(v)$ defined by

$$
G_{p r}(v)=\left(v E-A_{r}-\frac{1}{r}(r v+q)^{-d} A_{d}\right)^{-1}
$$

in which $A_{r}$ is given in (25).

Proof: Under the condition of the theorem, we can deduce that the uncertain discrete singular delay system (1) is regular, causal by using Lemma 4 and following a similar line as in the proof of Theorem 1. Next, we will establish that all the finite eigenvalues of system (1) are located within the disk $D_{\text {int }}(q, r)$. To this end, we note

$$
\operatorname{det}\left(v E-A_{r}-\frac{1}{r}(r v+q)^{-d} A_{d}\right) \neq 0, \quad|v| \geq 1
$$

and

$$
G_{p r}(v)=\sum_{k=0}^{\infty} G_{r}(k) v^{-k}
$$

It follows that

$$
\begin{aligned}
\rho\{ & \left.\frac{1}{r} G_{p r}(v)\left[\Delta A+(r v+q)^{-d} \Delta A_{d}\right]\right\} \\
& \leq \rho\left\{\frac{1}{r}\left|G_{p r}(v)\right|_{m}\left[|\Delta A|_{m}+|r v+q|^{-d}\left|\Delta A_{d}\right|_{m}\right]\right\} \\
& \leq \rho\left(\frac{1}{r} H_{r}\left(M_{A}+\delta^{-d} M_{d}\right)\right)<1
\end{aligned}
$$


for $|v| \geq 1$. Therefore, we have

$$
\operatorname{det}\left(I-r^{-1} G_{p r}(v)\left(\Delta A+(r v+q)^{-d} \Delta A_{d}\right)\right) \neq 0, \quad|v| \geq 1
$$

This together with (30) implies that

$$
\begin{aligned}
\operatorname{det} & {\left[v E-A_{r}-\frac{1}{r} \Delta A-\frac{1}{r}(r v+q)^{-d}\left(A_{d}+\Delta A_{d}\right)\right] } \\
= & \operatorname{det}\left(v E-A_{r}-\frac{1}{r}(r v+q)^{-d} A_{d}\right) \\
& \cdot \operatorname{det}\left(I-\frac{1}{r} G_{p r}(v)\left(\Delta A+(r v+q)^{-d} \Delta A_{d}\right)\right) \neq 0, \quad|v| \geq 1 .
\end{aligned}
$$

By Lemma 4, it follows from this inequality that

$$
\rho\left(E, A+\Delta A, A_{d}+\Delta A_{d}\right) \subset D_{\text {int }}(q, r) .
$$

This completes the proof.

Remark 2: The criteria developed in Theorems 1 and 2 are computationally simple, and can be tested easily. Moreover, it can be seen that all advantages stated in [3] and [17] are still preserved in our method to deal with uncertain discrete singular delay systems with structured uncertainties. It is also worth pointing out that the results in both Theorems 1 and 2 can be extended to the case of multiple time delays.

\section{NUMERICAL EXAMPLE}

In this section, we give an example to illustrate the effectiveness of the proposed method.

Consider the linear uncertain discrete singular delay system (1) with parameters as follows:

$$
\begin{aligned}
E & =\left[\begin{array}{lll}
1 & 0 & 0 \\
0 & 0 & 0 \\
0 & 1 & 0
\end{array}\right] \quad A=\left[\begin{array}{ccc}
0.1 & 0 & 0 \\
0 & 0 & -0.4 \\
0 & 0.3 & 0.5
\end{array}\right] \\
A_{d} & =\left[\begin{array}{ccc}
0.12 & 0.2 & 0 \\
0 & 0 & 0.1 \\
0 & 0.1 & -0.1
\end{array}\right] \quad M_{A}=\left[\begin{array}{ccc}
0.1 & 0.01 & 0.1 \\
0.01 & 0 & 0.01 \\
0 & 0.01 & 0.1
\end{array}\right] \\
M_{d} & =\left[\begin{array}{ccc}
0.03 & 0 & 0.1 \\
0 & 0.01 & 0.02 \\
0.01 & 0 & 0.1
\end{array}\right]
\end{aligned}
$$

and $d=1$. It can be verified that the nominal singular delay system is regular, causal, and the eigenvalues of the nominal system can be calculated as

$$
\sigma\left(E, A, A_{d}\right)=\{-0.3,-0.2,0.25,0.4,0.5\} .
$$

In this example, we set $D_{\text {int }}(q, r)=D_{\text {int }}(0.1,0.6)$. Thus, $\sigma\left(E, A, A_{d}\right) \subset D_{\text {int }}(0.1,0.6)$. The purpose of this example is to check whether the uncertain system is still regular, causal and the eigenvalues of the perturbed system are located within disk $D_{\text {int }}(0.1,0.6)$.

From (29), the matrix $H_{r}$ is computed as

$$
H_{r}=\left[\begin{array}{ccc}
1.6667 & 2.9461 & 1.5556 \\
0 & 3.2407 & 2.3334 \\
0 & 2.3333 & 0
\end{array}\right]
$$

Then, we have

$$
\begin{aligned}
\rho\left(r^{-1} H_{r}\left(M_{A}+\delta^{-d} M_{d}\right)\right) \\
=\rho\left(\left[\begin{array}{lll}
0.5454 & 0.1519 & 1.8567 \\
0.1318 & 0.1469 & 1.4367 \\
0.0389 & 0.0778 & 0.1944
\end{array}\right]\right)=0.8264<1 .
\end{aligned}
$$

Therefore, by Theorem 2, we have that the uncertain discrete singular delay system is still regular, causal and all its finite eigenvalues are located within disk $D_{\text {int }}(0.1,0.6)$ for all admissible uncertainties.

\section{CONCLUSIONS}

In this brief, we have developed a computationally simple approach to analyze the D-stability robustness for uncertain discrete singular systems with state delay. Sufficient conditions have been obtained to guarantee not only robust pole location within a specified disk but also regularity, causality of the uncertain singular system. Structured uncertainties have been discussed. The proposed method can be easily extended to analyze the D-stability robustness for discrete singular systems with multiple time delays.

\section{REFERENCES}

[1] D. J. Bender and A. J. Laub, "The linear-quadratic optimal regulator for descriptor systems," IEEE Trans. Automat. Control, vol. 32, pp. 672-688, Aug. 1987.

[2] C.-T. Chen, Linear System Theory and Design. New York: Holt Rinehart and Winston, 1984.

[3] J.-H. Chou, "Pole-assignment robustness in a specified disk," Syst. Contr. Lett., vol. 16, no. 1, pp. 41-44, 1991.

[4] D. Cobb, "Feedback and pole placement in descriptor variable systems," Int. J. Control, vol. 33, no. 6, pp. 1135-1146, 1981.

[5] L. Dai, Singular Control Systems. Berlin, Germany: Springer-Verlag, 1989.

[6] C.-H. Fang and L. Lee, "Robustness of regional pole placement for uncertain continuous-time implicit systems," IEEE Trans. Automat. Control, vol. 39, pp. 2303-2307, Nov. 1994.

[7] C.-H. Fang, L. Lee, and F.-R. Chang, "Robust control analysis and design for discrete-time singular systems," Automatica, vol. 30, no. 11, pp. 1741-1750, 1994.

[8] L. R. Fletcher, "Pole assignment and controllability subspaces in descriptor systems," Int. J. Control, vol. 66, no. 5, pp. 677-709, 1997.

[9] K. Furuta and S. Kim, "Pole assignment in a specified disk," IEEE Trans. Automat. Control, vol. 32, pp. 423-427, May 1987.

[10] G. Garcia and J. Bernussou, "Pole assignment for uncertain systems in a specified disk by state feedback," IEEE Trans. Automat. Control, vol. 40, pp. 184-190, Jan. 1995.

[11] A. Kumar and P. Daoutidis, "Feedback control of nonlinear differential-algebraic equation systems," AIChE J., vol. 41, no. 3, pp. 619-636, 1995.

[12] C. H. Lee, T. H. S. Li, and F. C. Kung, "D-stability analysis for discrete systems with a time delay," Syst. Control Lett., vol. 19, no. 3, pp. 213-219, 1992.

[13] F. L. Lewis, "A survey of linear singular systems," Circuits, Syst. Signal Processing, vol. 5, no. 1, pp. 3-36, 1986.

[14] R. W. Newcomb, "The semistate description of nonlinear time-variable circuits," IEEE Trans. Circuits Syst., vol. 28, pp. 62-71, Jan. 1981.

[15] L. Pandolfi, "Generalized control systems, boundary control systems and delayed control systems," Maths. Contr. Sig. Syst., vol. 3, no. 2, pp. $165-181,1990$.

[16] T.-J. Su and W.-J. Shyr, "Robust D-stability for linear uncertain discretedelay systems," IEEE Trans. Automat. Control, vol. 39, pp. 425-428, Feb. 1994.

[17] H. Trinh and M. Aldeen, "D-stability analysis of discrete-delay perturbed systems," Int. J. Control, vol. 61, no. 2, pp. 493-505, 1995.

[18] S. Xu and C. Yang, "Stabilization of discrete-time singular systems: A matrix inequalities approach," Automatica, vol. 35, no. 9, pp. 1613-1617, 1999. 